\title{
Computer simulations of block copolymer tethered nanoparticle self-assembly
}

\author{
Elaine R. Chan and Lin C. Ho \\ Department of Chemical Engineering, University of Michigan, Ann Arbor, Michigan 48109-2136 \\ Sharon C. Glotzer ${ }^{a}$ ) \\ Department of Chemical Engineering, University of Michigan, Ann Arbor, Michigan 48109-2136 \\ and Department of Materials Science and Engineering, University of Michigan, Ann Arbor, \\ Michigan 48109-2136
}

(Received 16 May 2006; accepted 5 July 2006; published online 9 August 2006)

\begin{abstract}
We perform molecular simulations to study the self-assembly of block copolymer tethered cubic nanoparticles. Minimal models of the tethered nanoscale building blocks (NBBs) are utilized to explore the structures arising from self-assembly. We demonstrate that attaching a rigid nanocube to a diblock copolymer affects the typical equilibrium morphologies exhibited by the pure copolymer. Lamellar and cylindrical phases are observed in both systems but not at the corresponding relative copolymer tether block fractions. The effect of nanoparticle geometry on phase behavior is investigated by comparing the self-assembled structures formed by the tethered NBBs with those of their linear $A B C$ triblock copolymer counterparts. The tethered nanocubes exhibit the conventional triblock copolymer lamellar and cylindrical phases when the repulsive interactions between different blocks are symmetric. The rigid and bulky nature of the cube induces interfacial curvature in the tethered NBB phases compared to their linear $A B C$ triblock copolymer counterparts. We compare our results with those structures obtained from $A B C$ diblock copolymer tethered nanospheres to further elucidate the role of cubic nanoparticle geometry on self-assembly. () 2006 American Institute of Physics. [DOI: 10.1063/1.2241151]
\end{abstract}

\section{INTRODUCTION}

Self-assembly is a promising means for designing and constructing precise nanostructures as precursors for materials with diverse applications. ${ }^{1}$ Soft materials such as block copolymers and surfactants can self-assemble into rich morphologies, e.g., micellar, cylindrical, gyroid, and lamellar structures, depending on molecular composition, the degree of incompatibility between the blocks, and, in the case of triblock and higher order multiblock copolymers, the sequence of the blocks in the copolymer. A wide variety of nanoscale building blocks (NBBs), including nanorods, ${ }^{2}$ nanocubes, ${ }^{3,4}$ and nanoplates, ${ }^{5}$ have been synthesized, and functionalization of these NBBs with synthetic macromolecules such as block copolymers provides new opportunities for creating nanoparticle/polymer assemblies with novel or enhanced properties. Advances in the syntheses of nanoparticles with macromolecular tethers attached ${ }^{6}$ have motivated investigations aimed at elucidating and understanding the self-assembly principles for these objects.

Zhang et al. ${ }^{7}$ recently proposed a computer simulation framework for modeling and predicting the self-assembly of tethered NBBs into ordered structures. ${ }^{8-11}$ They demonstrated that the thermodynamic immiscibility of the tethers and rigid nanoparticles, arising from conditions where the solvent is preferential for either one of those components, can lead to highly ordered nanostructures reminiscent of the

\footnotetext{
a) Author to whom correspondence should be addressed. Electronic mail: sglotzer@umich.edu
}

self-assembled phases observed in conventional block copolymer, surfactant, and liquid crystalline systems, as well as new phases uncommon to these traditional soft matter systems.

Several detailed theoretical and simulation studies of the self-assembled structures resulting from tethered NBBs composed of various nanoparticle shapes attached to a single polymeric chain have been undertaken. The structures have been compared with those arising from self-assembly of their linear block copolymer counterparts to gain insight on the effect of nanoparticle geometry and molecular composition on self-assembly. For example, Lee et al. ${ }^{12}$ used a combination of self-consistent field theory and density functional theory to investigate the self-assembly in two dimensions of "tadpole" molecules with a spherical nanoparticle head group and either a homopolymer ( $A B$ tadpole) or diblock copolymer ( $A B B$ tadpole) tail. They found that $A B$ tadpoles with composition $55 \%$ tail ( $A$ block) and $45 \%$ sphere $(B$ block) self-assemble into a hexagonally close-packed cylinder phase instead of a lamellar morphology that linear diblock copolymer melts would exhibit with the same molecular composition. However, tadpoles with a diblock copolymer tail and composition $60 \% \mathrm{~A}$ and $40 \% \mathrm{~B}$ exhibit a lamellar morphology. Iacovella et al. ${ }^{10}$ investigated the phase behavior of similar $A B$ monotethered nanospheres in three dimensions via Brownian dynamics simulations. They observed self-assembly of the NBBs into cubic micellar, hexagonal cylindrical, perforated lamellar, and lamellar phases, as observed in conventional linear chain surfactant systems, 
(a)

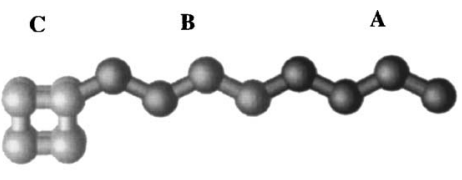

(b)

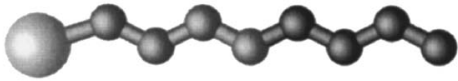

(c)

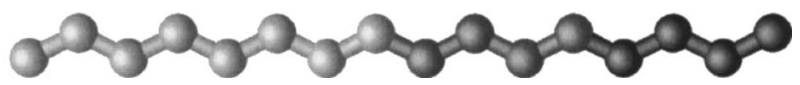

FIG. 1. Schematic representations of the simulation models investigated. Each NBB has a diblock copolymer tether attached to either a nanoparticle or its linear chain counterpart. (a) $A B C$ diblock copolymer tethered nanocube. (b) $A B C$ diblock copolymer tethered nanosphere. (c) Linear $A B C$ triblock copolymer.

depending on the nanosphere diameter, overall concentration, and temperature. Building blocks consisting of nanospheres with relatively small diameters exhibit a tendency to assemble into sheetlike morphologies, while those with larger nanospheres prefer to assemble into curved morphologies. Zhang et al. ${ }^{11}$ conducted a similar simulation study of $A B$ monotethered nanocube assemblies to model selfassembly in tethered polyhedral oligomeric silsesquioxane (POSS) systems at the mesoscale. They demonstrated that the nanocubes have a propensity to assemble into lamellar morphologies because of the cubic nanoparticle geometry.

Here we extend prior investigations of $A B$ tethered NBB self-assembly to that of diblock copolymer tethered nanoparticles ( $A B C$ tethered NBBs) and compare these structures with those formed by their linear $A B C$ triblock counterparts. This study is motivated by both the present capabilities to synthesize these molecules, ${ }^{13,14}$ as well as previous studies demonstrating that the morphological phase boundaries of conventional block copolymer systems change when the molecular architecture and conformation of the polymer coils are varied. ${ }^{15}$ These latter studies focused on self-assembly of polystrene-poly(benzyl ether) dendrimer-linear coil (PSdendr-PBE) diblock copolymers and reveal that differences in the architecture and conformation between the linear PS and dendritic PBE blocks induce shifts in the morphological phase boundaries toward higher compositional fractions of the linear block as compared to systems of linear diblock copolymers. Hence, these findings suggest that a linear polymer segment can be assembled into a lamellar morphology when it is the major component, and are thereby useful in designing self-assembling microphase-separated materials. We wish to investigate similar principles for molecules where a nanoparticle is introduced as one of the blocks instead of a polymeric block with a nonlinear architecture.

Presented herein are Brownian dynamics simulations of the self-assembly of a system of $A B C$ diblock copolymer tethered cubic NBBs (Fig. 1) into ordered nanostructures. We investigate how attachment of the cubic nanoparticle to the block copolymer influences the equilibrium assembled structures, and compare the results with the self-assembled microstructures observed in block copolymer and surfactant systems to gain further insight on the self-assembly behavior of these systems. The monotethered nanocube can be regarded as a minimal model representation of a POSS cube function- alized with a diblock copolymer, ${ }^{14}$ although it is also an applicable representation of other small cubic nanoparticles, such as platinum ${ }^{3}$ and gold and silver nanocubes, ${ }^{4}$ functionalized on one corner with a block copolymer. The phases exhibited by the tethered nanocubes are compared to those formed by flexible coil $A B C$ triblock copolymers and block copolymer tethered nanospheres to assess the influence of the cubic nanoparticle geometry on morphology as compared to the linear and spherical counterparts, respectively. The simulation approach is instructive for gaining an understanding of the assembly process and as a guide to understanding how parameters such as nanoparticle geometry can be manipulated to construct useful structures from self-assembly.

\section{SIMULATION MODEL AND METHOD}

Simplified minimal models of tethered nanocubes, tethered nanospheres, and linear block copolymers in implicit solvent are employed in the simulations. Figure 1 illustrates the simulation models used here and further details are reported elsewhere. ${ }^{7,8,10,11}$ To mimic immiscibility between different bead types, the interactions between them are specified as follows. If the solvent is selectively poor for one type of bead, then those beads interact via a truncated and shifted Lennard-Jones (LJ) potential, ${ }^{16}$

$$
\begin{aligned}
U_{\mathrm{LJ}}(r)= & 4 \varepsilon\left[\left(\frac{\sigma}{r}\right)^{12}-\left(\frac{\sigma}{r}\right)^{6}-\left(\frac{\sigma}{r_{c}}\right)^{12}+\left(\frac{\sigma}{r_{c}}\right)^{6}\right] \\
& -\left(r-r_{c}\right)\left(\frac{d U_{\mathrm{LJ}}(r)}{d r}\right)_{r=r_{c}}, \quad r \leqslant r_{c}
\end{aligned}
$$

where $r_{c}=2.5 \sigma$ is the cutoff distance beyond which the LJ interaction is set to zero and $r$ is the distance between two beads. This potential accounts for both excluded volume and van der Waals interactions. Conversely, if the solvent is selectively good for a particular bead type, then those beads interact via a purely repulsive Weeks-Chandler-Andersen ${ }^{17}$ (WCA) soft-sphere potential,

$$
U_{\mathrm{WCA}}(r)=4 \varepsilon\left[\left(\frac{\sigma}{r}\right)^{12}-\left(\frac{\sigma}{r}\right)^{6}\right]+\varepsilon, \quad r \leqslant r_{c},
$$

where $r_{c}=2^{1 / 6} \sigma$. Two different types of beads also interact via this purely excluded volume interaction to mimic immiscibility between them.

The block copolymer tethered NBBs can be regarded as analogues of $A B C$ linear triblock copolymer chains, where the nanoparticle represents one end block $C$ and the diblock copolymer tether comprises the middle block $B$ and end block $A$. There are three Flory-Huggins interaction parameters $-\chi_{A B}, \chi_{B C}, \chi_{A C}$ that influence the types of selfassembled morphologies possible here. The interactions between the different blocks can be symmetric (i.e., $\chi_{A B}=\chi_{B C}$ $=\chi_{A C}$ ) or asymmetric (i.e., $\chi_{A B}, \chi_{B C}$, and $\chi_{A C}$ not all equal), resulting in a rich variety of morphologies that have been predicted and observed in triblock and multiblock copolymer systems. ${ }^{18-22}$ For simplicity, we focus here on systems of tethered NBBs where the interactions between different blocks or bead types are symmetric. Hence, all pairs of dif- 
ferent species of beads experience the same repulsive strength or degree of immiscibility $\left(\varepsilon_{A B}=\varepsilon_{B C}=\varepsilon_{A C}\right)$.

Each block copolymer tether or chain is modeled as a collection of soft spheres or monomers connected by springs. In this bead-spring model, bonded monomers along a chain interact via an attractive finitely extensible nonlinear elastic ${ }^{23}$ (FENE) potential,

$$
U_{\mathrm{FENE}}(l)=\left\{\begin{array}{cc}
-0.5 k L_{0} \ln \left[1-\left(\frac{l}{L_{0}}\right)^{2}\right] & l<L_{0} \\
\infty & l \geqslant L_{0}
\end{array},\right.
$$

where $l$ is the distance between adjacent monomers and $k$ and $L_{0}$ are the energy and length parameters of the potential. As in prior works, the parameters are assigned the values $k$ $=30$ and $L_{0}=1.5 \sigma$ to ensure relatively stiff bonds while avoiding very high-frequency modes and chain crossing.

The Brownian dynamics simulation method is used to simulate self-assembly of the tethered NBBs and linear block copolymers in implicit solvent. ${ }^{24}$ Brownian dynamics is a stochastic molecular dynamics simulation method in which the equation of motion for each bead $i$ is

$$
m_{i} \dot{\mathbf{v}}_{i}(t)=-m_{i} \xi_{i} \mathbf{v}_{i}(t)+\mathbf{F}_{i}\left(\mathbf{x}_{i}(t)\right)+\mathbf{R}_{i}(t),
$$

where $m_{i}$ is the mass of bead $i$, and $\mathbf{x}_{i}, \mathbf{v}_{i}, \mathbf{F}_{i}$, and $\xi_{i}$ represent the position, velocity, force, and friction coefficient acting on bead $i$, respectively. ${ }^{25}$ The friction coefficient is assumed to be independent of position and time, and the stochastic force $\mathbf{R}_{i}$ has no correlation with the prior velocities or with the conservative force. These simulations sample the canonical ensemble, as the friction coefficient and stochastic noise term couple the system to a heat bath and effectively function together as a nonmomentum conserving thermostat.

Reduced units, where the basic units for length and energy are $\sigma$ and $\varepsilon$ of the LJ potential, respectively, are used. Each block copolymer tether bead and nanocube corner bead has the same size $\sigma=1$ and mass $m=1$. Each nanosphere has size $2 \sigma$ and mass $8 m$ to match the dimensions of a single nanocube. The results are presented in terms of reduced units so that the reduced temperature is defined as $T^{*}=k_{B} T / \varepsilon$, the interaction energy parameter as $\chi \sim \varepsilon^{*}=\varepsilon / k_{B} T$, the reduced time step as $\Delta t=t / \sigma \sqrt{m / \varepsilon}$, and the reduced friction coefficient as $\xi^{*}=\xi \sigma \sqrt{m / \varepsilon}$. The system concentration is based on the reduced number density of beads $\rho^{*}=\sum_{i} m_{i} N_{i} \sigma_{i}^{3} / V$, where $N_{i}$ denotes the number of particles of type $i$ (tether bead, cube corner bead, or nanosphere) and $V$ is the volume of the simulation cell.

Systems of $N b=1000$ tethered NBBs or block copolymer chains ( $N=16000$ total particles) are simulated using cubic simulation boxes and periodic boundary conditions at concentration $\rho^{*}=0.86$ and temperature $T^{*}=1$. The equations of motion are integrated using the leap-frog algorithm ${ }^{16}$ with time step $\Delta t=0.01$. The value of the friction coefficient is specified as $\xi^{*}=1$ for each particle so that the simulations occur between the overdamped and purely deterministic regimes. The rigid-body motion of the cubes is captured using the method of quaternions. ${ }^{16}$ Each system is initially relaxed athermally (i.e., excluded volume interactions only) and then cooled gradually to the target temperature to prevent the sys-
TABLE I. Self-assembled structures for tethered nanocubes under various solvent conditions.

\begin{tabular}{lccc}
\hline \hline \multicolumn{1}{c}{$f_{B}{ }^{\text {a }}$} & $\begin{array}{c}\text { Selective good } \\
\text { solvent for cubes }\end{array}$ & $\begin{array}{c}\text { Neutral poor } \\
\text { solvent }\end{array}$ & $\begin{array}{c}\text { Selective poor } \\
\text { solvent for cubes }\end{array}$ \\
\hline 0 & Lamellae & Lamellae & Lamellae \\
0.125 & Lamellae & Lamellae & Lamellae \\
0.25 & Lamellae & Lamellae & Lamellae \\
0.375 & $\begin{array}{c}\text { hcp core-shell } \\
\text { cylinders } \\
\text { hcp core-shell } \\
\text { cylinders }\end{array}$ & Lamellae & Lamellae \\
0.50 & Lamellae & Lamellae \\
0.625 & $\begin{array}{c}\text { hcp core-shell } \\
\text { cylinders } \\
\text { hcp core-shell } \\
\text { cylinders }\end{array}$ & Lamellae & Lamellae \\
0.75 & Lamellae & Lamellae & Lamellae \\
0.875 & Lamellae \\
\hline \hline
\end{tabular}

${ }^{\mathrm{a}}$ Relative volume fraction of the $B$ block on the diblock copolymer tether.

tems from getting trapped into metastable states. Because nonequilibrium structures can depend on the cooling history, both the size of the temperature increments at which the initial athermal configurations are cooled to the target temperature, as well as the system sizes $N b$, are varied to verify that the same equilibrium structures form. The structural evolution of each system is monitored as a function of time by inspecting snapshots of configurations.

\section{RESULTS AND DISCUSSION}

\section{A. Tethered nanocube self-assembly}

We first present and discuss simulation results for the diblock copolymer tethered nanocube assemblies. The phase behavior of these systems under three different implicit solvent conditions is investigated here: (1) selectively good solvent for the cubes (i.e., cubes are solvophilic) and poor solvent for the tethers (i.e., tethers are solvophobic), where the interactions between cube beads are of the WCA type and the interactions between $A$ tether beads $(A-A)$ and between $B$ tether beads $(B-B)$ are each of the LJ type; (2) neutrally poor solvent, where the interactions between the same species of beads $(A-A, B-B, C-C)$ are each of the LJ type; and (3) selectively poor solvent for the cubes and good solvent for the tethers, where the interactions between cube beads are of the $\mathrm{LJ}$ type and the $A-A$ and $B-B$ tether bead interactions are each of the WCA type.

\section{Selective good solvent for nanocubes}

The interactions between nanocubes are modeled as purely excluded volume under this solvent condition to assess the effect of simply attaching the rigid cubic nanoparticle to the copolymer on morphological behavior. Table I summarizes the self-assembled structures formed over a range of $B$ block fractions $\left(f_{B}\right)$ from 0 to 0.875 in the diblock copolymer tether. The ranges of the relative $B$ block fractions are defined here as the following: low $f_{B}=0-0.25$, intermediate $f_{B}=0.375-0.625$, and high $f_{B}=0.75-0.875$.

The tethered nanocubes self-assemble into lamellar structures when the $B$ block component constitutes a low or high content in the copolymer tether [Fig. 2(a)]. At interme- 


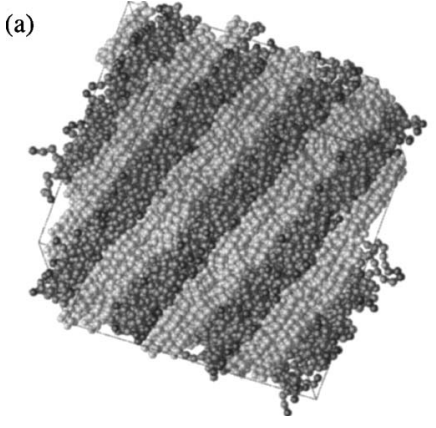

(b)

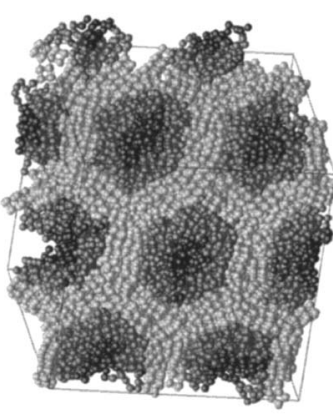

FIG. 2. Simulation snapshots of equilibrium morphologies of tethered nanocubes in selectively good solvent for the cube. (a) Tethered nanocubes self-assemble into lamellae at $f_{B}=0.875$. (b) Tethered nanocubes with $f_{B}$ $=0.75$ self-assemble into hexagonally arranged core-shell cylinders with the $A$ block (black) comprising the core, the $B$ block (dark gray) the shell, and the $C$ block nanocubes (light gray) the matrix.

diate and high values of the $B$ block fraction, when the composition of the copolymer tether is equal or nearly equal with respect to the $A$ and $B$ blocks, the tethered nanocubes exhibit core-shell cylindrical structures consisting of $A$ cores and $B$ shells in a matrix composed of nanocubes [Fig. 2(b)]. The cylindrical structures are arranged in a hexagonal pattern. This type of morphology differs from those displayed in conventional diblock copolymer systems at the corresponding values of relative block volume fraction. We also compare this morphology with those obtained from flexible coil triblock copolymers in Sec. B below.

Comparing the equilibrium morphologies between the tethered nanocube system and linear diblock copolymer melts, the presence of the rigid cube appears to preclude assembly of the NBBs into spherical micelles and influences assembly into lamellar and hexagonally arranged cylindrical phases at relative copolymer tether block fractions that are significantly different from the block fractions that give rise to such structures in the linear copolymer systems. Specifically, we find that structures with flat interfaces (lamellae) occur at low and high values of $f_{B}$, while structures with curved interfaces (micelles and cylinders) are typically present at these values in diblock copolymer melts. We also notice that at intermediate values of $f_{B}$, the tethered nanocubes display curved morphologies (core-shell cylinders), while linear diblock copolymers tend to exhibit flat lamellar phases.

The different morphologies arising in the tethered nanocube systems can be understood by considering the preference for interfacial curvature ${ }^{22,26}$ in these NBBs and the local intermolecular packing of the cubes. Lamellae are typically observed in linear block copolymer systems when the block compositions in the copolymer are equal or close to equal. This phase results because of the similarity in the conformations of the blocks. However, when a rigid cube is attached to one end of the copolymer, the bulkiness of the nanoparticle can be expected to increase the amount of stretching of the $B$ block (now the middle block) of the molecule in the lamellar phase. To alleviate the increases in stretching energy, the interfaces between the bulky cube and the $B$ block, and between the $B$ block and the $A$ block, become curved away from the larger volume component to form the observed core-shell cylindrical structures. Hence, core-shell cylindrical structures are observed in the tethered nanocube systems when the copolymer tether block fractions are equal or nearly equal. We compute the end-to-end distances for the $A$ and $B$ blocks on the tethers in this system to confirm that these blocks are not stretched in the equilibrium cylindrical structures. The distance values here are nearly equal to those in the corresponding system without cubes. The repulsive interactions between cubes also discourage local face-to-face packing of the cubes, and the absence of this local intermolecular packing appears to disfavor selfassembly into lamellar phases under this particular solvent condition.

The simulations also predict that the tethered nanocubes self-assemble into lamellar structures when the composition of one of the copolymer tether blocks is very small. When the relative fraction of one of the copolymer blocks is zero, the case of nanocubes functionalized with homopolymers is recovered. Zhang et al. previously showed that these tethered NBBs exhibit a tendency to self-assemble into lamellae. ${ }^{11}$ Lamellar phases typically are not observed in linear diblock copolymer systems at the low relative block fraction that these phases are found for the tethered nanocubes. For tethered nanocubes with small $f_{B}$, lamellar structures occur because the $B$ block on the copolymer chain is now attached to both the cube and the linear $A$ block. The connectivity of the minor $B$ block to these two components forces it to mix with the cube-rich and $A$-rich layers. There is no preference here for the $B$ block to mix with one type of lamellar sheet because the strength of the $A B$ and $B C$ repulsions are equal $\left(\chi_{A B}=\chi_{B C}=\chi_{A C}\right)$. Simulation snapshots reveal that the $B$ block is distributed randomly at the interface between the cube-rich and A-rich layers. A similar mixing of the minor middle block component in the majority block phases was also observed in two-dimensional mean field calculations of linear $A B C$ triblock copolymer phase behavior. ${ }^{18}$ For tethered nanocubes with small $f_{A}$, lamellar morphologies appear consisting of cube-rich and $B$-rich layers with the minor $A$ block present at the interface [Fig. 2(a)]. This result is not surprising because the composition of this NBB approaches that of a homopolymer functionalized cube. There is no preference for the $A$ block to mix with one type of lamellar sheet because of the symmetry of the interactions between different bead types.

To obtain a more rigorous understanding of how attaching a nanocube to a linear diblock copolymer affects the resulting equilibrium morphologies of the tethered nanocubes, we proceeded to remove the cubes in the tethered NBB self-assembled structures and simulate assembly of only the diblock copolymer tethers or chains in the resulting dilute solution. We find that the latter systems exhibit lamellar phases in the simulations at intermediate values of relative block volume fractions. However, when one of the block volume fractions is low, macrophase separation into solventrich and disordered copolymer-rich phases is observed instead of the conventional spherical micelle and hexagonal cylinder phases. The macrophase separation can be attributed to the attractive interactions between tether beads ${ }^{27}$ and the much smaller concentration of chains $\left(\rho^{*}=0.43\right.$ after the 


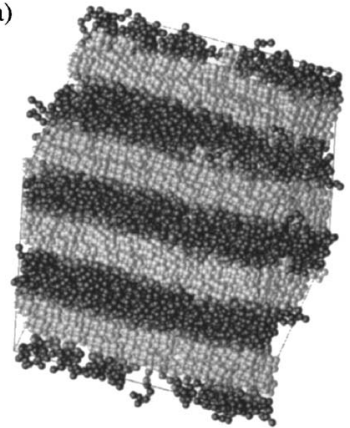

(b)

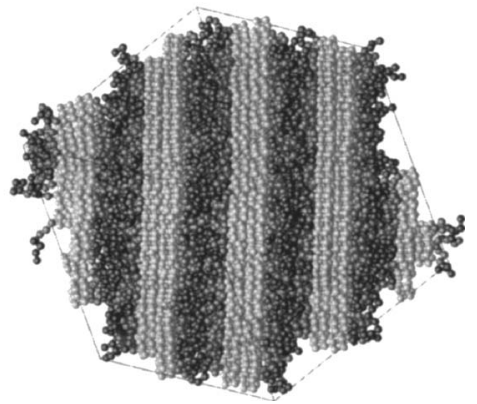

FIG. 3. Lamellar phases observed in the tethered nanocube systems. (a) In neutral poor solvent and at $f_{B}=0.25$, the lamellae exhibit an $A B C C B A$ sequence. (b) In selective poor solvent and at $f_{B}=0.5$, the $A$ and $B$ blocks mix in the layers. A liquid crystalline ordering of the cubes is evident (light gray layers).

cubes are removed) at temperature $T^{*}=1$. When the dilute systems are heated to higher temperatures, a transition from the phase separated system to a disordered homogeneous solution of chains is observed.

Although a direct comparison between the phases exhibited by the block copolymer tethered nanocubes and by homopolymer functionalized monotethered nanocubes from a previous simulation study ${ }^{11}$ cannot be made here because of the difference in system concentrations, it is interesting to note that the results for concentrated homopolymer functionalized nanocube systems are in agreement with the morphology-concentration trend observed for monotethered nanospheres in selective solvent good for spheres from simulation. ${ }^{10}$ The latter systems undergo transitions from curved micellar and cylindrical structures to flat lamellar morphologies with increasing concentration. Zhang et al. previously demonstrated that nanocubes with a single homopolymer tether form cylindrical phases at moderate concentration $\rho^{*}=0.57$, and we find that our higher concentrated systems form lamellar phases. These results suggest that the phase diagram predicted for homopolymer functionalized monotethered nanospheres may be applicable for predicting trends in the mesoscopic phase behavior of systems of monotethered cubes and other NBB shapes, although not necessarily with respect to local packing of the particles. ${ }^{9}$

\section{Neutral poor solvent for nanocubes and tethers}

In the second case where the solvent is neutrally poor for each bead type, we find that the tethered nanocubes selfassemble into solely lamellar phases over the entire range of relative tether block fractions studied. Figure 3(a) shows a representative simulation snapshot at relative diblock copolymer tether volume fraction $f_{B}=0.25$. Unlike the lamellae formed in the selectively good solvent conditions for the cubes, an $A B C C B A$ sequence of sheets is observed here. This pattern has been predicted and observed in linear triblock copolymer melts ${ }^{19}$ and is captured in the tethered nanocube systems by the interactions in the model; $A-A, B-B$, and $C$ - $C$ interactions are each of the attractive LJ type here. We also find that the cubes are packed in a regular face-to-face manner and hence exhibit liquid crystalline ordering. ${ }^{28}$ The

TABLE II. Self-assembled structures of linear $A B C$ triblock copolymers under various solvent conditions.

\begin{tabular}{|c|c|c|c|}
\hline$f_{B}{ }^{\mathrm{a}}$ & $\begin{array}{c}\text { Selective good } \\
\text { solvent for } C \text { block }\end{array}$ & $\begin{array}{c}\text { Neutral poor } \\
\text { solvent }\end{array}$ & $\begin{array}{c}\text { Selective poor } \\
\text { solvent for } C \text { block }\end{array}$ \\
\hline 0 & Lamellae & Lamellae & Lamellae \\
\hline 0.125 & Lamellae & Lamellae & Lamellae \\
\hline 0.25 & Lamellae & Lamellae & Lamellae \\
\hline 0.375 & $\begin{array}{l}\text { hcp core-shell } \\
\text { cylinders }\end{array}$ & Lamellae & Lamellae \\
\hline 0.50 & Perforated lamellae & Lamellae & Lamellae \\
\hline 0.625 & Perforated lamellae & Lamellae & Lamellae \\
\hline 0.75 & $\begin{array}{l}\text { hcp core-shell } \\
\text { cylinders }\end{array}$ & Lamellae & Lamellae \\
\hline 0.875 & Lamellae & Lamellae & Lamellae \\
\hline
\end{tabular}

$\overline{\bar{a} \text { Relative volume fraction of the } B \text { block on the diblock copolymer tether. }}$

absence of cylindrical phases in the neutral poor solvent conditions suggests that the local face-to-face intermolecular packing of the cubes strongly stabilizes lamellar structures, as was demonstrated in a recent simulation study of homopolymer tethered nanocube self-assembly. ${ }^{11}$

\section{Selective poor solvent for nanocubes}

The simulations predict self-assembly into exclusively lamellar morphologies under this solvent condition. These findings are similar to the neutral poor solvent results. However, inspection of simulation snapshots reveals that the lamellae in these systems do not exhibit the $A B C C B A$ sequence of sheets. A simulation snapshot from a system where $f_{B}=0.5$ is shown in Fig. 3(b). Although the cubes are organized in a well-defined face-to-face pattern within cube-rich layers, the tether beads form layers in which the $A$ blocks and $B$ blocks are mixed. These results arise as a consequence of the nature of the interactions between blocks, and demonstrate that the composition of the layers in the lamellar morphology can be tuned by varying the solvent conditions (solvophility/solvophobicity) for the different components of the tethered NBB.

\section{B. Linear $A B C$ triblock copolymer self-assembly}

We now turn our attention to how the cubic geometry of the nanoparticle affects self-assembly compared to the compositionally equivalent linear counterpart. Replacing the cube by its linear counterpart roughly results in a conventional $A B C$ triblock copolymer where the $C$ end block is comprised of eight beads. The equilibrium self-assembled structures of the triblock copolymers simulated under various solvent conditions are summarized in Table II and described below. Low, intermediate, and high values of the $B$ block fraction $f_{B}$ are defined in the same manner as in the previous section.

\section{Selective good solvent for the linear $C$ end block}

In the case where the linear $C$ end block is solvophilic and the copolymer tether $A$ and $B$ blocks are solvophobic, the simulations reveal lamellar phases at block fraction values $f_{B}=0-0.25$ and 0.875 , while core-shell cylindrical phases are predicted at relative block fraction values $f_{B}$ 
(a)

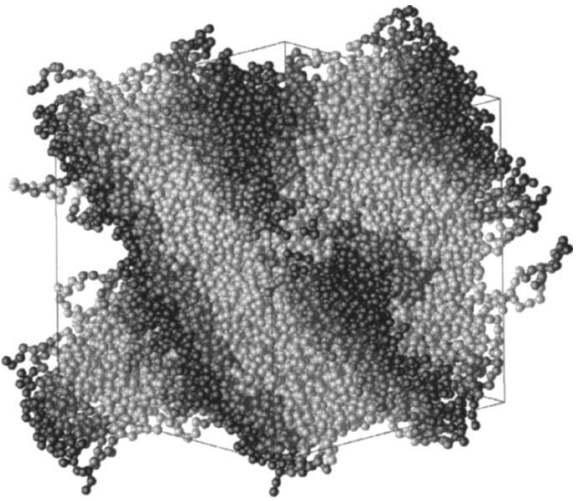

(b)

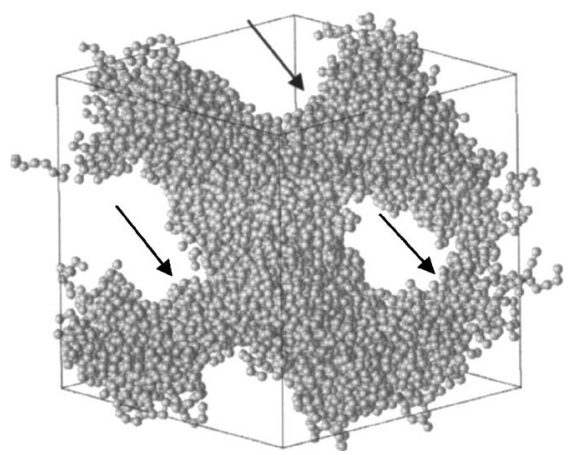

FIG. 4. Simulation snapshots of a self-assembled perforated lamellar phase in a linear $A B C$ triblock copolymer system where the solvent is selectively good for the $C$ block and $f_{B}=0.625$. (a) Lamellae composed of $A$ and $B$ tether blocks and of solely $C$ end blocks are observed. (b) The tether blocks are removed to illustrate the $C$ end block bridges, denoted by arrows, that puncture the tether-rich layers and form holes in those layers.

$=0.375$ and 0.75 . At intermediate block fraction values $f_{B}$ $=0.5-0.625$, perforated lamellar phases are observed in which layers composed of $A$ and $B$ tether beads are punctured by the $C$ end blocks, thereby forming holes in the lamellae. Representative simulation snapshots of this phase are shown in Fig. 4.

The simulations predict that cylindrical morphologies occur over a range of intermediate and high values of relative $B$ block fraction in the tethered nanocube system, but in only two of the linear triblock copolymer systems. The curved morphologies for the latter may arise from the constraint that the linear $C$ end block is compositionally equivalent to the cube (i.e., same $f_{C}$ value), which does not necessarily translate to geometric equivalence between the linear segment and the cube. In fact, the $C$ block is swollen because of the selective good solvent conditions and consequently, the radius of gyration of the linear $C$ segment is larger than both the effective radius (one half the body diagonal) of the cube and the radius of gyration of the adjacent $A B$ copolymer segment. Previous simulation studies of amphiphilic tethered nanospheres and surfactants ${ }^{10}$ indicate that curved morphologies are preferred when the volume of the head group exceeds that of the tail group. ${ }^{29}$ This trend may be applicable to the systems here if we loosely regard the $C$ end block of the triblock copolymer chain as the head group and the adjacent $A B$ copolymer segment as the tail group.

Although the radius of gyration of the $C$ block is larger than that of the $A B$ copolymer segment over the entire range of $f_{B}$ studied, the simulations predict lamellar instead of cylindrical structures for low relative $B$ block fractions $f_{B}$ $=0.125-0.25$. Cylindrical structures may not be observed in these systems because of the low content of the $B$ middle block in the tethers; the low $f_{B}$ values correspond to only one and two $B$ monomers on the tether, respectively. It is possible that there are simply not enough $B$ monomers on each tether to wrap around the $A$ cores to form a cylindrical shell. The attractive interactions between the $B$ monomers may also not be strong enough to promote formation of curved morphologies because the number of $B$ monomers is small compared to the numbers of $A$ and $C$ monomers. Both of these situations can be attributed to the short tether lengths studied here to satisfy the constraint that both the $C$ block segment and the nanocube have equal composition, i.e., both have the same number of $C$ beads.

The perforated lamellae predicted by the simulations at intermediate $B$ block fractions $f_{B}=0.5-0.625$ may be attributed to a complex combination of factors. This phase has an interfacial curvature that is intermediate to those of lamellar and cylindrical structures. The short tether lengths could preclude the formation of cylindrical phases in these systems for the reasons discussed above. A lamellar morphology for the tethers may be favored because the relative amounts of the $A$ and $B$ blocks are equal or nearly equal. However, perforations in the layers may occur in our simulations due to the intricate interplay between interfacial tension and formation of domains with uniform thickness. ${ }^{30}$ In diblock copolymer melts, constant mean curvature (CMC) surfaces are favored in order to minimize interfacial area and chain stretching favors domains of uniform thickness. Complex phases such as perforated lamellae arise in these systems because a CMC interface produces large variations in the domain thickness, and consequently the interfaces deviate from $\mathrm{CMC}$ to achieve more uniform domain sizes. Perhaps such a competition occurs in the simulated self-assembly studies of our model triblock copolymers, which each consist of a majority $C$ end block that is swollen due to the solvophilic interactions between these bead types in the model, leading to the perforated lamellar structures observed here.

To further investigate the effect of geometry on selfassembly between the linear triblock copolymer and tethered nanocube systems, we also perform simulations of "modified triblock chains," defined here as copolymers where the $C$ block length is reduced to four or five monomers so that the radius of gyration of the block is approximately equal to the effective radius (one half the body diagonal) of the nanocube. We find that these modified triblock copolymers exhibit a propensity to self-assemble into lamellae. Compared to the tethered nanocube systems, the modified triblock chains do not form curved cylindrical morphologies. These findings suggest that the presence of the rigid cubic nanoparticle induces curvature in the tethered nanocube morphologies.

\section{Neutral poor solvent for all linear blocks}

When the solvent condition is modified to be neutrally poor for the linear triblock copolymer segments, the simulations predict morphologies that consist of three-layer lamel- 
(a)

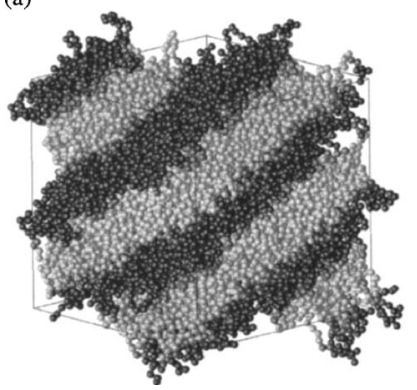

(b)

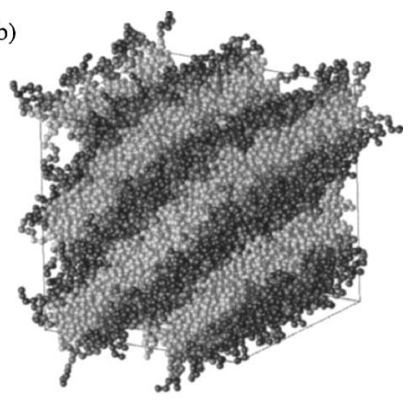

FIG. 5. Simulation snapshots of self-assembled linear $A B C$ triblock copolymer systems. (a) In neutral poor solvent, lamellae in an $A B C C B A$ pattern are observed $\left(f_{B}=0.25\right)$. (b) In selective poor solvent the lamellae do not display an $A B C C B A$ pattern $\left(f_{B}=0.5\right)$. The $C$-rich layers do not exhibit liquid crystalline ordering compared to the tethered nanocube system [Fig. 3(b)].

lae where the copolymers assemble into sheets that stack on top of each other in the order $A B C C B A$ due to the connectivity of the three blocks. Figure 5(a) illustrates this morphology for a system where $f_{B}=0.25$. This phase is in agreement with theoretical calculations of linear $A B C$ triblock copolymer phase behavior, where it was demonstrated that lamellar phases prevail when one of the end blocks in the triblock copolymer has relative compositional fraction of $0.5\left(f_{C}\right.$ $=0.5$ here) and the strength of the interactions between unlike blocks are equal or symmetric. ${ }^{18,20}$ Although both the tethered nanocubes and the linear triblock copolymers selfassemble into solely lamellar structures under this solvent condition, a fundamental difference exists in the local intermolecular packing of the $C$-rich layers in both systems. The $C$-rich layers exhibit no clear ordering of monomers within them in the triblock copolymer chain systems, while the analogous layers in the tethered nanocube systems consist of cubes packed in a distinct face-to-face manner and display liquid crystalline ordering. Hence, diblock copolymer tethered nanocubes could be useful for producing functional devices comprised of the rich three-layer $A B C C B A$ lamellae typically formed by linear triblock copolymers, but with an additional liquid crystalline ordering in the cube-rich sheets.

\section{Selective poor solvent for the linear C end block}

Under this solvent condition where the $C$ end block is solvophobic and the $A$ and $B$ blocks of the copolymer tether are solvophilic, the linear triblock copolymer chains tend to self-assemble into lamellar structures over the entire range of $f_{B}$ investigated. The lamellae do not display the $A B C C B A$ pattern because of mixing of the $A$ and $B$ blocks of the copolymer tether in alternating layers that arises from the symmetry of the repulsive interactions between unlike blocks [Fig. 5(b)]. Similar to the neutral solvent systems described above, simulation snapshots do not reveal any local ordering of monomers within the $C$-rich layers.

\section{Tethered nanosphere self-assembly}

Finally, it is interesting to examine how the geometry of a cubic nanoparticle affects self-assembly compared to a spherical nanoparticle shape. The focus here is on selected
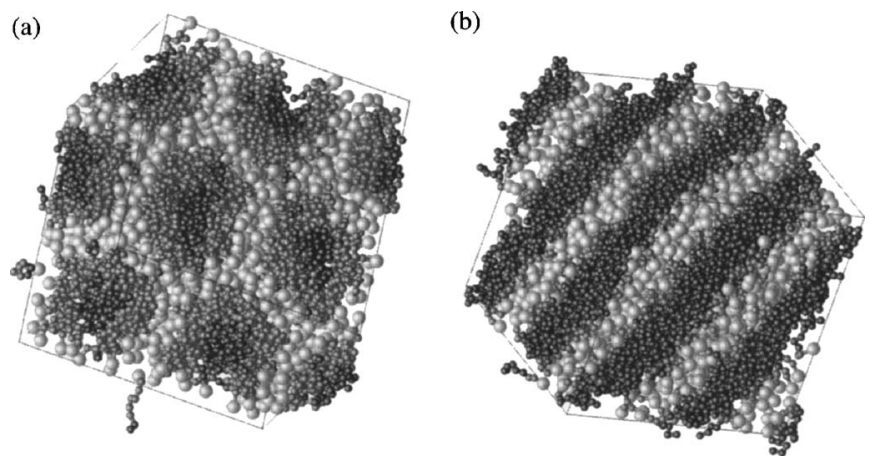

FIG. 6. Simulation snapshots of diblock copolymer tethered nanosphere systems. (a) In selective good solvent, core-shell cylinders are observed at $f_{B}=0.75$. (b) In neutral poor solvent, lamellae in an $A B C C B A$ pattern are observed $\left(f_{B}=0.25\right)$. Unlike the tethered nanocube systems [Fig. 3(a)], the spheres comprising the $C$-rich layers do not display distinct local packing or ordering.

cases investigated above but with the cube now replaced by a sphere. The nanospheres have a diameter that is twice that of each tether monomer and equal to the length of the nanocube body diagonal.

For the case where the solvent is selectively good for the nanoparticle and poor for the tether, self-assembly of diblock copolymer tethered nanospheres with $f_{B}=0.75$ is examined. The simulations predict assembly of the tethered NBBs into core-shell cylinders arranged in a hexagonal pattern [Fig. 6(a)]. This phase behavior is also observed in simulations of the compositionally equivalent tethered nanocubes and linear triblock copolymer chains. Although nanoparticle shape does not appear to significantly influence the type of morphologies formed under these solvent conditions, this property does affect the shape of the individual cylinders. The cylindrical structures in the tethered nanosphere systems have a round cross section, while those formed by the tethered nanocubes have a distinct hexagonal cross section [Fig. 2(b)]. The hexagonal shaped cylinders have flat edges that are promoted by the flat faces of the rigid cubes. Further conclusion can be made that the rigidity of the cubic or spherical nanoparticle induces curvature in the assembled structures, because the cylindrical morphology differs from the lamellar structures observed in the simulations of the modified (geometrically equivalent) linear triblock copolymers.

To investigate the effect of changing the composition of the polymer tether on phase behavior of the tethered NBBs, the simulated structures of this diblock copolymer tethered nanosphere are compared with those predicted for the corresponding homopolymer functionalized nanospheres from simulation. ${ }^{10}$ The simulations reveal that under the same system conditions (temperature, concentration, and solvent selectivity), the diblock copolymer tethered nanospheres assemble into core-shell cylindrical structures while the homopolymer tethered nanospheres assemble into lamellar sheets. Hence, the composition of the polymer tether can influence the type of morphology formed in the tethered nanosphere systems.

Additional tethered nanosphere systems of varying $f_{B}$ and solvent quality are examined. In neutral poor solvent, 
lamellar phases arise for low and intermediate relative $B$ block fractions $\left(f_{B}=0.125-0.375\right)$ and the sheets exhibit an $A B C C B A$ pattern [Fig. 6(b)]. Although the tethered nanocubes also display this precise morphology at the corresponding $f_{B}$ values, there is a difference in the local packing of spheres and cubes in the $C$-rich layers. The liquid crystalline ordering arising from the face-to-face local packing of cubes within the sheets (Fig. 3) is absent in the corresponding tethered nanosphere structures. At high relative $B$ block fractions $f_{B}=0.75-0.875$, the simulations predict core-shell cylinder morphologies. This phase differs from the lamellae observed in the tethered nanocube systems and further supports the notion that the face-to-face local packing of the cubes stabilizes lamellar structures.

\section{CONCLUSION}

Brownian dynamics simulations were utilized to investigate the self-assembly of nanocubes with a single diblock copolymer tether attached at one corner. Simplified minimal models of the tethered NBBs were used that capture the essential elements of connectivity and interaction specificity capable of predicting structures for a broad class of tethered NBB systems. These models facilitate access to the long length and time scales pertinent to the assembly process while foregoing atomistic detail. The influence of solvent quality on the resulting morphologies was obtained. The geometry of the rigid cube was found to induce self-assembly of the tethered NBBs into both lamellar and cylindrical phases when the cubes were solvophilic and the tethers were solvophobic. Structures with flat interfaces (lamellae) were predicted at low and high values of the tether block fraction $f_{B}$, while structures with curved interfaces (micelles and cylinders) are typically present at these values in diblock copolymer melts. At intermediate values of $f_{B}$, the tethered nanocubes displayed curved morphologies (core-shell cylinders), while linear diblock copolymers tend to exhibit flat lamellar phases. In neutral poor solvent and selective solvent that was poor for the cubes and good for the tethers, only lamellar structures were observed. In these latter systems, the cubes were observed to pack in a face-to-face manner and exhibit liquid crystalline ordering. The local intermolecular packing appears to promote and stabilize the sheet structures in these systems.

The morphologies of the tethered nanocubes were compared with those of their compositionally linear $A B C$ triblock copolymer counterparts to gain insight on the influence of nanocube geometry. Both systems exhibited lamellar morphologies at the same $f_{B}$ when the solvent was neutrally poor for all blocks or selectively poor for the cubes and good for the tethers. Lamellar, cylindrical, and perforated lamellar phases were observed when the solvent was instead selectively good for the linear $C$ end block and poor for the tethers. The short tether lengths were surmised to be responsible in part for the variety of structures found in some of the systems. Simulations of modified linear triblock copolymers where the radius of gyration of the $C$ end block was equal to the effective radius of the nanocube suggested that nanopar- ticle geometry plays a significant role in inducing curvature in the self-assembled tethered NBB morphologies for certain solvent conditions.

Additional comparisons were performed by replacing the cubic nanoparticles with their spherical counterparts in a few selected systems. It was further established that the geometry of the nanoparticle encourages assembly into curved structures under selective good solvent conditions for the nanoparticle, and we demonstrated that the composition of the polymer tether influences the types of self-assembled structures possible. Although the shape of the nanoparticle does not appear to affect the type of assembled morphology obtained in this solvent, it does influence the cross-sectional shape of the individual cylinders. The morphologies of the tethered nanospheres were altered compared to their tethered nanocube counterparts upon changing the solvent type to neutrally poor at high values of $f_{B}$.

\section{ACKNOWLEDGMENTS}

We thank P. T. Cummings, X. Zhang, and Z. L. Zhang for helpful suggestions, and are grateful to C. R. Iacovella and M. A. Horsch for assistance with the tethered nanosphere simulation code. The University of Michigan Center for Advanced Computing is acknowledged for support of our computer cluster. Financial support for this work has been provided by the National Science Foundation under Grant No. DMR-0103399.

${ }^{1}$ S. C. Glotzer, M. J. Solomon, and N. A. Kotov, AIChE J. 50, 2978 (2004).

${ }^{2}$ B. D. Busbee, S. O. Obare, and C. J. Murphy, Adv. Mater. (Weinheim, Ger.) 15, 414 (2003).

${ }^{3}$ T. S. Ahmadi, Z. L. Wang, T. C. Green, A. Henglein, and M. A. El-Sayed, Science 272, 1924 (1996).

${ }^{4}$ Y. G. Sun and Y. N. Xia, Science 298, 2176 (2002).

${ }^{5}$ T. D. Clark, R. Ferrigno, J. Tien, K. E. Paul, and G. M. Whitesides, J. Am. Chem. Soc. 124, 5419 (2002).

${ }^{6}$ S. Westenhoff and N. A. Kotov, J. Am. Chem. Soc. 124, 2448 (2002); B. S. Kim and P. T. Mather, Macromolecules 35, 8378 (2002); W. J. Parak, T. Pellegrino, C. M. Micheel, D. Gerion, S. C. Williams, and A. P. Alivisatos, Nano Lett. 3, 33 (2003); T. Song, S. Dai, K. C. Tam, S. Y. Lee, and S. H. Goh, Langmuir 19, 4798 (2003).

${ }^{7}$ Z. L. Zhang, M. A. Horsch, M. H. Lamm, and S. C. Glotzer, Nano Lett. 3, 1341 (2003).

${ }^{8}$ E. R. Chan, X. Zhang, C.-Y. Lee, M. Neurock, and S. C. Glotzer, Macromolecules 38, 6168 (2005).

${ }^{9}$ M. A. Horsch, Z. L. Zhang, and S. C. Glotzer, Phys. Rev. Lett. 95, 056105 (2005).

${ }^{10}$ C. R. Iacovella, M. A. Horsch, Z. L. Zhang, and S. C. Glotzer, Langmuir 21, 9488 (2005).

${ }^{11}$ X. Zhang, E. R. Chan, and S. C. Glotzer, J. Chem. Phys. 123, 184718 (2005).

${ }^{12}$ J. Y. Lee, A. C. Balazs, R. B. Thompson, and R. M. Hill, Macromolecules 37, 3536 (2004).

${ }^{13}$ J. Pyun, K. Matyjaszewski, T. Kowalewski, D. Savin, G. Patterson, G. Kickelbick, and N. Huesing, J. Am. Chem. Soc. 123, 9445 (2001).

${ }^{14}$ G. Cardoen, X. Hu, D. Baskaran, J. W. Mays, S. P. Gido, T. P. Russell, and E. B. Coughlin, Polym. Prepr. (Am. Chem. Soc. Div. Polym. Chem.) 46, 783 (2005)

${ }^{15}$ M. E. Mackay, Y. Hong, M. Jeong, B. M. Tande, N. J. Wagner, S. Hong, S. P. Gido, R. Vestberg, and C. J. Hawker, Macromolecules 35, 8391 (2002); D. J. Pochan, L. Pakstis, E. Huang, C. J. Hawker, and R. Vestberg, ibid. 35, 9239 (2002).

${ }^{16}$ M. P. Allen and D. J. Tildesley, Computer Simulation of Liquids (Clarendon, Oxford, 1987).

${ }^{17}$ J. D. Weeks, D. Chandler, and H. C. Andersen, J. Chem. Phys. 54, 5237 (1971). 
${ }^{18}$ W. Zheng and Z. G. Wang, Macromolecules 28, 7215 (1995).

${ }^{19}$ M. J. Ko, S. H. Kim, and W. H. Jo, Macromol. Theory Simul. 10, 38 (2001); Fibers Polym. 3, 8 (2002); Fibers Polym. 4, 15 (2003).

${ }^{20}$ P. Tang, F. Qiu, H. D. Zhang, and Y. L. Yang, Phys. Rev. E 69, 031803 (2004)

${ }^{21}$ Y. Mogi, H. Kotsuji, Y. Kaneko, K. Mori, Y. Matsushita, and I. Noda, Macromolecules 25, 5408 (1992); A. I. Triftaridou, M. Vamvakaki, C. S. Patrickios, and L. Lue, Macromol. Symp. 183, 133 (2002); R. Wang, J. L. Hu, Z. B. Jiang, and D. S. Zhou, Macromol. Theory Simul. 14, 256 (2005).

${ }^{22}$ S. P. Gido, D. W. Schwark, E. L. Thomas, and M. D. Goncalves, Macromolecules 26, 2636 (1993).

${ }^{23}$ G. S. Grest and K. Kremer, Phys. Rev. A 33, 3628 (1986).
${ }^{24}$ M. A. Horsch, Z. L. Zhang, C. R. Iacovella, and S. C. Glotzer, J. Chem. Phys. 121, 11455 (2004).

${ }^{25}$ W. F. van Gunsteren, H. J. C. Berendsen, and J. A. C. Rullmann, Mol. Phys. 44, 69 (1981); G. S. Grest, M. D. Lacasse, K. Kremer, and A. M. Gupta, J. Chem. Phys. 105, 10583 (1996).

${ }^{26}$ S. P. Gido and Z. G. Wang, Macromolecules 30, 6771 (1997).

${ }^{27}$ C. I. Huang and T. P. Lodge, Macromolecules 31, 3556 (1998).

${ }^{28}$ B. S. John, A. Stroock, and F. A. Escobedo, J. Chem. Phys. 120, 9383 (2004).

${ }^{29}$ J. N. Israelachvili, Intermolecular and Surface Forces (Academic, London, 1992).

${ }^{30}$ M. W. Matsen and F. S. Bates, Macromolecules 29, 7641 (1996); J. Chem. Phys. 106, 2436 (1997). 